\title{
Endovascular treatment of pseudoaneurysms in pancreatitis
}

\author{
Maciej Czernik, Ludomir Stefańczyk, Wojciech Szubert, Jarosław Chrząstek, Marcin Majos, Piotr Grzelak, Agata Majos \\ Department of Radiology - Diagnostic Imaging, Medical University of Lodz, Poland
}

Videosurgery Miniinv 2014; 9 (2): 138-144

DOI: $10.5114 /$ wiitm.2014.41621

\begin{abstract}
Introduction: Haemorrhagic complications are commonly reported in the course of acute pancreatitis (AP). The most dangerous are pseudoaneurysms secondary to wall erosion of the vessels crossing the inflammation area.

Aim: To evaluate the efficacy of different embolisation techniques of pseudoaneurysms secondary to AP.

Material and methods: The treatment outcomes in 10 AP patients were analysed in a follow-up period of 1 to 10 months (most frequently: splenic or gastroduodenal artery aneurysms). A total of 12 endovascular procedures were performed (thrombin embolisation, coil embolisation or combined). The efficacy of aneurysm sac exclusion and the patency of the treated vessel were compared.

Results: The follow-up examination on postprocedural day 7 revealed aneurysm refilling in 3 out of 11 cases. Three patients showed an occlusion of the aneurysm-parent vessel. One month after the procedures, involving 10 patients, a vessel occlusion was confirmed in 3 and aneurysm sac refilling in another 2 patients. Taking into consideration the type of the procedure performed, treatment failure in the form of sac refilling was observed in half of thrombin-based procedures, $25 \%$ of coil embolisations and 33\% of sandwich technique procedures. Similar failure distribution was noted for vessel occlusion.

Conclusions: It seems that thrombin injection was least successful, whereas thrombin injection into the sac and implantation of coils into the aneurysm neck seems to be the optimal procedure, in respect to both the cost-effectiveness of the procedure and late outcomes.
\end{abstract}

Key words: pancreatitis, pseudoaneurysm, embolisation.

\section{Introduction}

Haemorrhagic complications are commonly reported in the course of acute pancreatitis (AP) among other serious problems such as necrosis or large fluid collections forming in the retroperitoneal space [1]. The most dangerous are pseudoaneurysms secondary to wall erosion of the vessels crossing the inflammation area [2-4]. They require immediate intervention as retroperitoneal, peritoneal and gastrointestinal bleeding pose a life-threatening condition leading to hypotension and shock [5, 6]. Due to many problems associated with open surgical treatment, interventional radiology procedures are being applied more widely although with high care [7-15].

\footnotetext{
Aim

The aim of this study was to evaluate the efficacy of different embolisation techniques of visceral vessel pseudoaneurysms secondary to AP.

\section{Material and methods}

The treatment outcomes of 10 patients with pseudoaneurysms secondary to clinically and laboratory-confirmed AP were analysed. The interventional
}

Address for correspondence:

Agata Majos MD, PhD, Department of Radiology - Diagnostic Imaging, Medical University of Lodz, 22 Kopcińskiego St, $90-153$ Lodz, Poland, phone: +48 504294 077, fax: +48 4267867 34, e-mail: agata.majos@umed.lodz.pl 
procedures were performed in the Department of Radiology-Diagnostic Imaging of the Medical University of Lodz in 2006-2013. The patient group consisted of 2 women and 8 men aged 31 to 66 years old, in whom it was possible to perform follow-up examinations within 1 month after the procedure. The pseudoaneurysms were diagnosed by computed tomography (CT) and Doppler ultrasound (USG-CD). Six patients had both imaging modalities performed. The CT-angiography was performed in 7 out of 10 patients. The formation of a pseudoaneurysm was a complication of the first episode of AP in 6 patients, whereas it was diagnosed in 4 other patients after exacerbation of the earlier pathological process concerning the pancreas. The most commonly affected vessels were the splenic artery (5/10 cases) and gastroduodenal artery (3/10). The CT-based evaluation of aneurysm diameter showed that it ranged from $28 \mathrm{~mm}$ to $70 \mathrm{~mm}$ (including the mural thrombus).

Once the diagnosis was established, each patient had angiography and an endovascular intervention planned. A total of 12 procedures were performed (4 coil embolisations, 4 thrombin injections, 3 sandwich technique procedures involving thrombin injection and coil embolisation, and 1 covered stent implantation). The type of procedure was determined by the availability of embolisation material and the ability to perform selective catheterisation of the aneurysmparent vessel. The operator aimed at excluding the aneurysm using as few embolisation coils as possible (a sandwich technique was preferred) and maintaining the patency of the vessel itself, especially large vessels such as the superior mesenteric artery or the aorta, whereas the occlusion of the splenic artery was accepted. The routine follow-up included US-CD on postprocedural day 7 and/or CT performed one month after the procedure. The evaluated parameters included the refilling of the aneurysm sac and the patency of the aneurysm-parent vessel. Table I presents the detailed epidemiological data.

\section{Results}

The follow-up examination on postprocedural day 7 revealed aneurysm refilling in 3 out of 11 cases ( 2 thrombin injections and 1 sandwich procedure). Therefore, 2 supplementary embolisation interventions were performed (Photos 1 A-D). Three patients showed an occlusion of the aneurysm-parent vessel (2 splenic arteries and 1 gastroduodenal artery). One month after the procedures, involving 10 patients (2 re-embolisation cases performed after the first follow-up examination were excluded), a vessel occlusion was confirmed in 3 and aneurysm sac refilling in another 2 patients. Taking into consideration the type of procedure performed, treatment failure in the form of sac refilling was observed in half of thrombin-based procedures, $25 \%$ of coil embolisations and $33 \%$ of sandwich technique procedures. Similar failure distribution was noted for vessel occlusion (i.e. half of thrombin-based procedures, $25 \%$

Table I. Materials

\begin{tabular}{|c|c|c|c|c|c|c|}
\hline $\begin{array}{l}\text { Patient } \\
\text { no. }\end{array}$ & $\begin{array}{l}\text { Initials } \\
\text { [age] }\end{array}$ & Gender & Inflammation & $\begin{array}{l}\text { Provided } \\
\text { exams }\end{array}$ & $\begin{array}{c}\text { Localisation of the } \\
\text { perforation }\end{array}$ & $\begin{array}{c}\text { The diameter of } \\
\text { extravasation } \\
\text { (together with the clot) }\end{array}$ \\
\hline 1 & $A C(65)$ & $\mathrm{F}$ & 2 & $\mathrm{CT}$ & $A O R$ & $60(70)$ \\
\hline 2 & $\mathrm{AA}(50)$ & $M$ & 1 & US/CT & $\mathrm{SL}$ & $33(50)$ \\
\hline 3 & $\mathrm{OM}(56)$ & $M$ & 1 & $\mathrm{CT}$ & SMA & $35(40)$ \\
\hline 4 & RS (44) & $M$ & 1 & $\mathrm{CT}$ & GDA & $44(54)$ \\
\hline 5 & WG (36) & $M$ & 2 & US-CD/CT & $S L$ & $30(44)$ \\
\hline 6 & ST (31) & $M$ & 3 & US-CD/CT & $S L$ & $22(47)$ \\
\hline 7 & WP (38) & $M$ & 1 & US-CD/CT & GDA & $35(45)$ \\
\hline 8 & נر (48) & $M$ & 3 & US-CD/CT & $S L$ & $20(28)$ \\
\hline 9 & WA (39) & $M$ & 1 & $\mathrm{CT}$ & SL & $40(56)$ \\
\hline 10 & BN (32) & $\mathrm{F}$ & 1 & US-CD/CT & GDA & $10(33)$ \\
\hline
\end{tabular}

$A O R$ - aorta, SL - splenic artery, SMA - superior mesenteric artery, GDA - gastroduodenal artery 

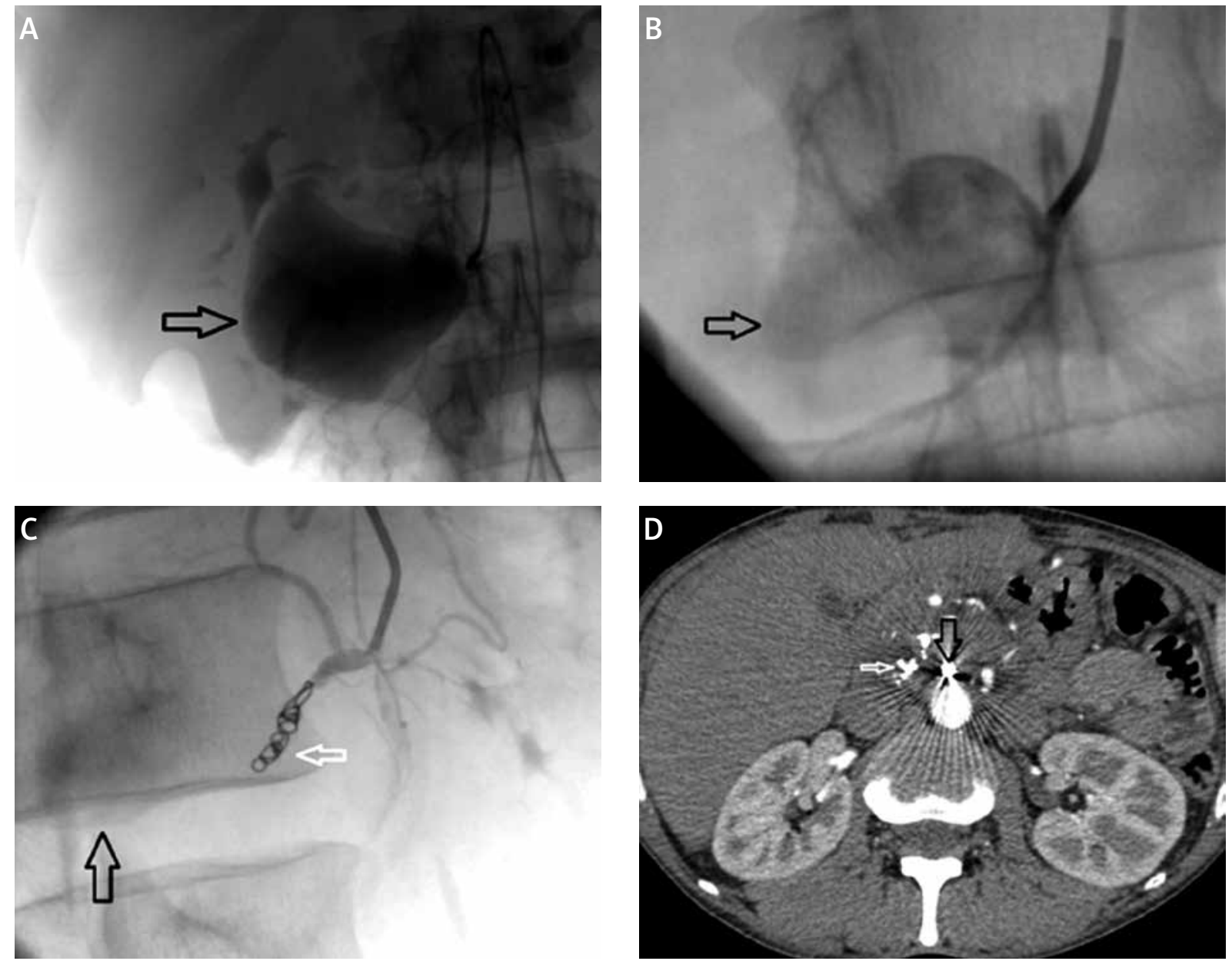

Photo 1. Patient RS - pseudoaneurysms of the gastroduodenal artery. A - Angiogram prior to thrombin injection. Contrast within the aneurysm sac (arrow). B - Angiogram prior to repeated thrombin injection leakage of contrast into the aneurysm sac secondary to thrombus recanalisation (arrow). C - Angiogram after coil embolisation (white arrow), successful aneurysm exclusion. Contrast trapped in the aneurysm sac (black arrow). D - CT image obtained during follow-up examination 1 month after the procedure. No aneurysm refilling is visible. The embolisation coil position is marked (black arrow). Note the post-inflammatory calcifications in the pancreas (white arrow)

of coil embolisations and 33\% of sandwich technique procedures, Photos 2 A-D). No significant organ complications of the performed procedures, which would require surgical intervention, were observed. Spleen ischemia secondary to occlusion of the splenic artery was observed in $\mathrm{CT}$ as evolving areas of limited post-contrast enhancement (Photos 3 A, B). Table II presents detailed data on treatment outcomes.

\section{Discussion}

The formation of pseudoaneurysms in chronic pancreatitis is associated with two different mechanisms, namely an intensive inflammatory process in the peripancreatic tissues and necrosis secondary to the release of proteolytic and lipolytic enzymes. The necrosis destroys the vessel wall, leading to its perforation and haemorrhage. The vessel wall is additionally damaged by bacterial infection and abscesses in the peripancreatic tissues, as well as long-lasting pseudocysts in the chronic phase of inflammation [2-4]. Apart from pancreatic enzymes the vessel wall may also be damaged by ischemia and compression $[3,4]$. The vessels most frequently affected by pseudoaneurysm include the splenic artery (40\%), gastroduodenal artery (20-30\%), pancreaticoduodenal artery (20-30\%) and others (5\%) [4, 6]. Injury to the 

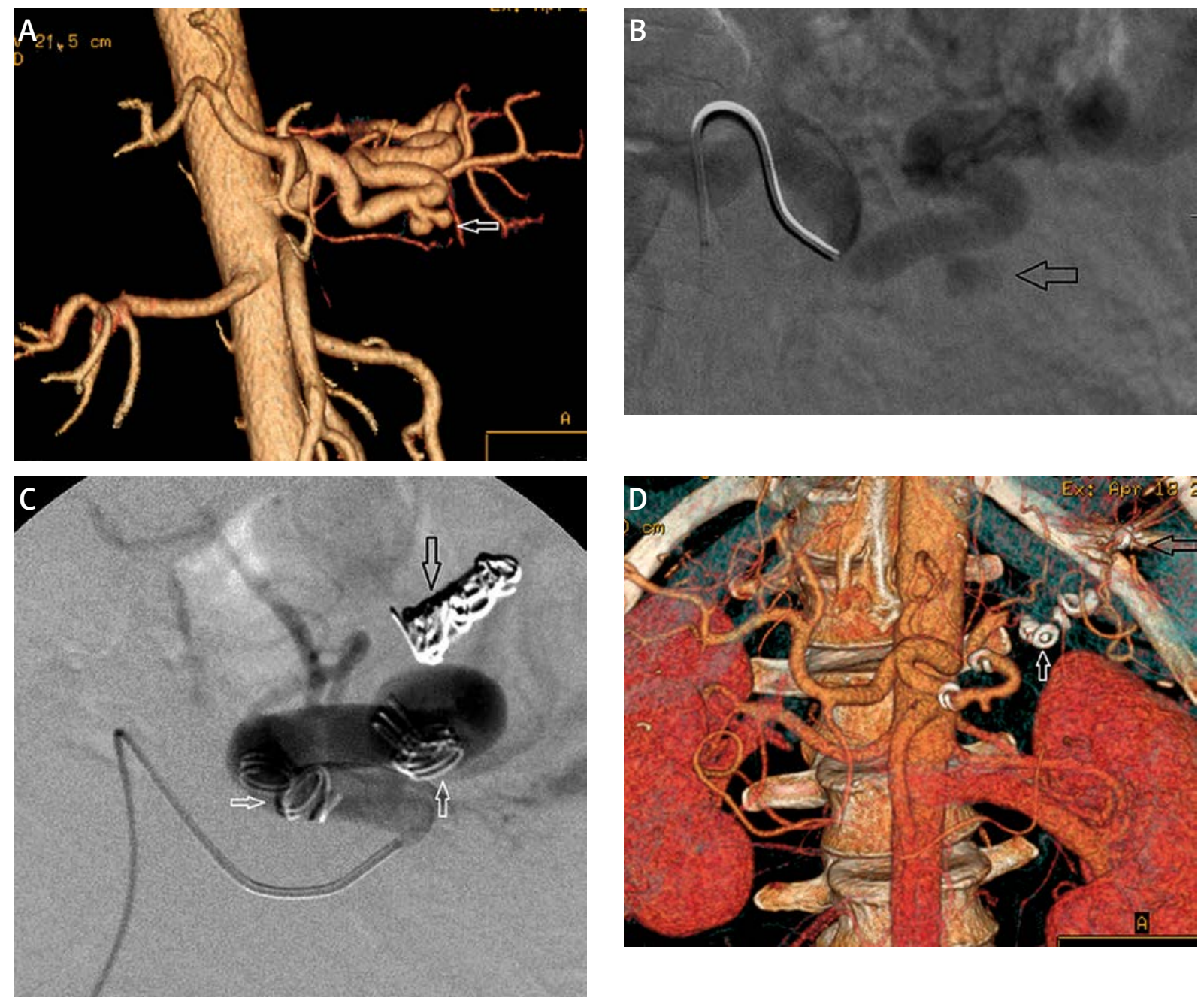

Photo 2. Patient JJ - pseudoaneurysms of the splenic artery. A - CT-angiography, 3D reconstruction of the aorta and visceral arteries. Contrast within small dual-chamber aneurysm of the proximal segment of the splenic artery (white arrow). B - Angiogram prior to embolisation - leakage of contrast into the aneurysm sac (arrow) is visible. The wide and tortuous splenic artery crosses the area over the lesion. C - Angiogram after embolisation. Coils were placed inside the aneurysm sac - successful aneurysm exclusion (black arrow). Other coils inside the splenic artery (white arrows). D - CT-angiography image obtained during follow-up examination 1 month after the procedure. No aneurysm refilling is visible. The embolisation coil position is marked (black arrow). No flow is registered in the distal segment of the splenic artery (white arrow)

aortic wall, included in the analysis, is a very rare condition and has already been reported $[8,16]$.

For many years ligation of the aneurysm artery was the sole treatment method in patients with pseudoaneurysms of visceral arteries, which was a severe trauma for patients with acute inflammation undergoing surgery [2]. The technological progress enabled endovascular procedures to be performed as an alternative to classic surgery [17]. Nowadays, embolisation of visceral artery aneurysms is commonly recognised as an optimal treatment modality. The sole indication for open surgery is the presence of a large pancreatic pseudocyst accompanying the pseudoaneurysm $[6,7]$. The most commonly used method of interventional radiology is coil embolisation [7-11], and the implantation of covered stents if larger and life-important arteries are affected $[14,16,18]$. The rate of technically successful embolisation ranges from $83 \%$ to $100 \%$ [12]. Apart from coils, which are used in over $80 \%$ of reported procedures, embolisation may also be performed using cyanoacrylates ( $\mathrm{n}-\mathrm{BCA}-\mathrm{N}$-butyl-cyanoacrylate), Spongostan or thrombin [9-13]. 

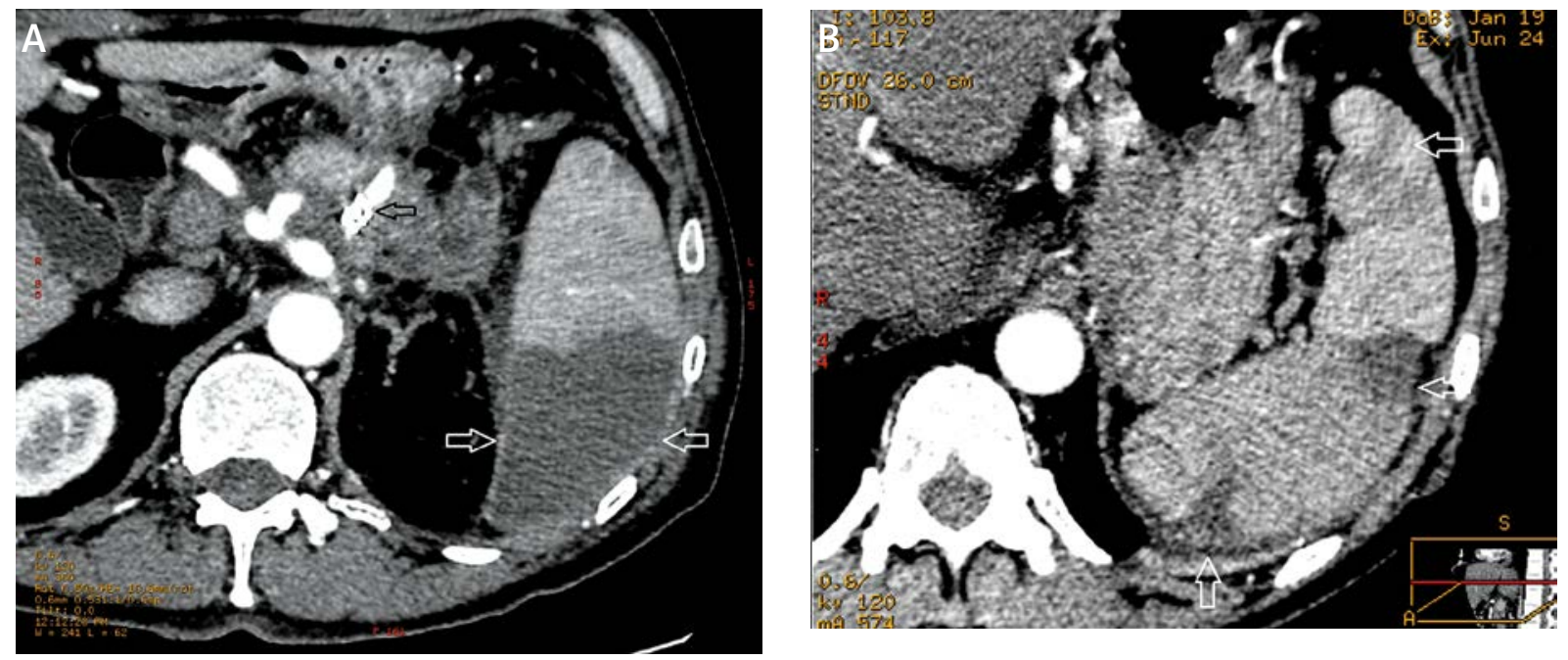

Photo 3. Patient J - status after embolisation of pseudoaneurysms of the splenic artery. A - CT-angiography image (axial plane). No contrast enhancement in the anterior pole of the spleen, secondary to the spleen artery occlusion (white arrows). B - CT-angiography image obtained during follow-up examination 1 month after the procedure. The spleen artery remains occluded. Hypoperfusion areas are reduced and separated within the spleen parenchyma (white arrows)

The most frequently reported method in the literature is proximal embolisation occluding the aneurysm-parent vessel, aneurysm sac embolisation or the so-called isolation technique, which consists of occlusion of parent and outflow vessels. It is of great importance in case of an active inflammato- ry process, as it allows the introduction of the embolisation material more distantly from the centre of the inflammatory process [9-11]. The combined "sandwich" technique includes the use of several embolisation materials. It is beneficial in cases of very large aneurysms, as it limits the number of coils

Table II. Results

\begin{tabular}{|c|c|c|c|c|c|c|c|c|}
\hline \multirow[t]{2}{*}{$\begin{array}{l}\text { Patent } \\
\text { no. }\end{array}$} & \multirow[t]{2}{*}{$\begin{array}{l}\text { Initials } \\
\text { [age] }\end{array}$} & \multirow[t]{2}{*}{ Gender } & \multirow[t]{2}{*}{$\begin{array}{l}\text { Spirals } \\
\text { (number) }\end{array}$} & \multirow[t]{2}{*}{$\begin{array}{l}\text { Biothrombin } \\
{[\mathrm{ml}]}\end{array}$} & \multicolumn{2}{|c|}{$\begin{array}{c}\text { Results on the } \\
\text { postprocedural day } 7\end{array}$} & \multicolumn{2}{|c|}{ Results after 1 month } \\
\hline & & & & & Patency & Refilling & Patency & Refilling \\
\hline 1 & AC (66) & $\mathrm{F}$ & SG & - & Yes & No & Yes & No \\
\hline 2 & $\mathrm{AA}(50)$ & M & 4 & - & Yes & No & Yes & No \\
\hline \multirow[t]{2}{*}{3} & OM (56) & M & 28 & 6 & Yes & Yes & - & - \\
\hline & & & 12 & - & Yes & No & Yes & Yes \\
\hline \multirow[t]{2}{*}{4} & RS (44) & M & - & 6 & Yes & Yes & - & - \\
\hline & & & 1 & - & Yes & No & Yes & No \\
\hline 5 & WG (36) & M & - & 6 & - & - & No & No \\
\hline 6 & ST (31) & M & - & 6 & No & No & Yes & No \\
\hline 7 & WP (38) & M & 7 & - & No & No & No & No \\
\hline 8 & J (48) & M & 10 & 14 & No & No & No & No \\
\hline 9 & WA (39) & M & 1 & 6 & Yes & No & Yes & No \\
\hline 10 & BN (32) & F & - & 2 & Yes & Yes & Yes & Yes \\
\hline
\end{tabular}


being used, which reduces the procedure costs, as well as the risk of compression injury as the evolution of inflammation takes place and bacterial infection lasts longer [9]. The possible proteolysis of the thrombus formed after thrombin injection does not raise the risk of aneurysm refilling, as long as the blood inflow from the parent artery is cut off by the coils [13]. Observations concerning the regression of aneurysms, whose presence was confirmed angiographically, are not uncommon. It results from successful mechanisms of haemostasis $[5,6$, 12]. Therefore, it may be assumed that the patient may benefit even from "time-limited" embolisation achieved by thrombin injection in the acute inflammatory phase, even if the medicament-induced thrombus should undergo proteolysis. The presented material proves the efficacy of the procedure performed using only thrombin, which was done in tortuous, narrow vessels difficult for catheterisation. It most commonly affected the splenic artery, whose occlusion, in the general opinion, does not lead to severe organ complications. Should a large and life-important vessel be treated, whose patency must be maintained (aorta, superior mesenteric artery), the endovascular intervention consists of the implantation of a covered stent. The implantation is then a life-saving procedure, yet the inflammatory process in the vicinity of the stent remains active, and the late outcome is not clear [16].

The limitation that applies to this manuscript is the small number of patients included, which rules out a more detailed statistical analysis. Due to the differentiated clinical picture, low diagnosis rate of this complication, many endovascular techniques applied and high mortality associated with the basic disease, the collection of comparable material remains problematic. Acute pancreatitis develops frequently in alcohol-dependant, non-compliant patients, which additionally hampers the follow-up of such patients, paradoxically, when the treatment outcome is good. The confirmation of the following conclusions requires large, multicentre studies.

\section{Conclusions}

Thrombin injection seems to be the least effective method for the treatment of pseudoaneurysms secondary to AP. Thrombin injection into the aneurysm sac and application of embolisation coils into its neck seems to be the optimal method in respect of the procedure cost-effectiveness. The occlusion of the splenic artery in our material did not cause any significant complications associated with organ ischemia.

\section{References}

1. Sileikis $A$, Beiša $\vee$, Beiša $A$, et al. Minimally invasive retroperitoneal necrosectomy in management of acute necrotizing pancreatitis. Videosurgery Miniinv 2013; 8: 29-35.

2. Hagiwara A, Miyauchi H, Shimazaki S. Predictors of vascular and gastrointestinal complications in severe acute pancreatitis. Pancreatology 2008; 8: 211-8.

3. Kirby J, Vora P, Midia M, et al. Vascular complications of pancreatitis: imaging and intervention. Cardiovasc Intervent Radiol 2008; 31: 957-70.

4. Mallick IH, Winslet MC. Vascular complications of pancreatitis. JOP 2004; 5: 328-37.

5. de Perrot M, Berney T, Bühler L, et al. Management of bleeding pseudoaneurysms in patients with pancreatitis. Br J Surg 1999; 86: 29-32.

6. Balachandra S, Siriwardena AK. Systematic appraisal of the management of the major vascular complications of pancreatitis. Am J Surg 2005; 190: 489-95.

7. Sethi H, Peddu P, Prachalias A, et al. Selective embolization for bleeding visceral artery pseudoaneurysms in patients with pancreatitis. Hepatobiliary Pancreat Dis Int 2010; 9: 634-8.

8. Kalva SP, Yeddula K, Wicky S, et al. Angiographic intervention in patients with a suspected visceral artery pseudoaneurysm complicating pancreatitis and pancreatic surgery. Arch Surg 2011; 146: 647-52.

9. Lau KY, Wong TP, Wong WW, et al. Transcatheter embolisation of visceral pseudoaneurysms: technical difficulties and modification of embolisation technique. Eur J Vasc Endovasc Surg 2005; 30: 133-6.

10. Tulsyan N, Kashyap VS, Greenberg RK, et al. The endovascular management of visceral artery aneurysms and pseudoaneurysms. J Vasc Surg 2007; 45: 276-83.

11. Ikeda O, Nakasone Y, Tamura Y, et al. Endovascular management of visceral artery pseudoaneurysms: transcatheter coil embolization using the isolation technique. Cardiovasc Intervent Radiol 2010; 33: 1128-34.

12. Spiliopoulos S, Sabharwal T, Karnabatidis D, et al. Endovascular treatment of visceral aneurysms and pseudoaneurysms: longterm outcomes from a multicenter European study. Cardiovasc Intervent Radiol 2012; 35: 1315-25.

13. Parildar M, Oran I, Memis A. Embolization of visceral pseudoaneurysms with platinum coils and N-butyl cyanoacrylate. Abdom Imaging 2003; 28: 36-40.

14. Rossi M, Rebonato A, Greco L, et al. Endovascular exclusion of visceral artery aneurysms with stent-grafts: technique and longterm follow-up. Cardiovasc Intervent Radiol 2008; 31: 36-42.

15. Synowiec T, Warot M, Burchard P, et al. Hemoptysis as a first symptom of endoleak after thoracic endovascular aortic repair, which caused aortic rupture and required complex management. Videosurgery Miniinv 2013; 8: 178-81. 
16. Stefańczyk L, Elgalal MT, Chrząstek J, et al. Pancreatitis-related abdominal aortic pseudoaneurysms treated with stent-grafts. Ann Vasc Surg 2012; 26: 730.e7-11.

17. Synowiec T, Chęciński P, Samolewski P, et al. Hybrid procedure in a patient with symptomatic thoraco-abdominal aneurysm and prior abdominal aortic reconstruction - case report. Videosurgery Miniinv 2012; 7: 132-6.

18. Poncyljusz W, Sagan L, Safranow K, et al. Initial experience with implantation of novel dual layer flow-diverter device FRED. Videosurgery Miniinv 2013; 8: 258-64.

Received: 19.09.2013, accepted: 5.11.2013. 